\title{
Analysis of Regional Mechanics in Canine Lung Injury Using Forced Oscillations and 3D Image Registration
}

\author{
David W. Kaczka, ${ }^{1,2}$ Kunlin CaO, ${ }^{3}$ Gary E. Christensen, ${ }^{3}$ Jason H. T. Bates, ${ }^{4}$ and Brett A. Simon ${ }^{1,2}$ \\ ${ }^{1}$ Harvard Medical School, Boston, MA, USA; ${ }^{2}$ Department of Anesthesiology, Critical Care, and Pain Medicine, Beth Israel \\ Deaconess Medical Center, 330 Brookline Avenue, Dana 717A, Boston, MA 02215, USA; ${ }^{3}$ University of Iowa, Iowa City, IA, \\ USA; and ${ }^{4}$ University of Vermont, Burlington, VT, USA
}

(Received 2 August 2010; accepted 19 November 2010; published online 4 December 2010)

Associate Editor Kenneth R. Lutchen oversaw the review of this article.

\begin{abstract}
Acute lung injury is characterized by heterogeneity of regional mechanical properties, which is thought to be correlated with disease severity. The feasibility of using respiratory input impedance $\left(Z_{\mathrm{rs}}\right)$ and computed tomographic (CT) image registration for assessing parenchymal mechanical heterogeneity was evaluated. In six dogs, measurements of $Z_{\mathrm{rs}}$ before and after oleic acid injury at various distending pressures were obtained, followed by whole lung CT scans. Each $Z_{\mathrm{rs}}$ spectrum was fit with a model incorporating variable distributions of regional compliances. CT image pairs at different inflation pressures were matched using an image registration algorithm, from which distributions of regional compliances from the resulting anatomic deformation fields were computed. Under baseline conditions, average model compliance decreased with increasing inflation pressure, reflecting parenchymal stiffening. After lung injury, these average compliances decreased at each pressure, indicating derecruitment, alveolar flooding, or alterations in intrinsic tissue elastance. However, average compliance did not change as inflation pressure increased, consistent with simultaneous recruitment and strain stiffening. Image registration revealed peaked distributions of regional compliances, and that small portions of the lung might undergo relative compression during inflation. The authors conclude that assessments of lung function using $Z_{\mathrm{rs}}$ combined with the structural alterations inferred from image registration provide unique but complementary information on the mechanical derangements associated with lung injury.
\end{abstract}

Keywords-Acute lung injury, Dog, Computed tomography, Respiratory mechanics, Input impedance.

\section{INTRODUCTION}

Acute lung injury (ALI) is characterized by respiratory failure in the presence of airway closure and

Address correspondence to David W. Kaczka, Department of Anesthesiology, Critical Care, and Pain Medicine, Beth Israel Deaconess Medical Center, 330 Brookline Avenue, Dana 717A, Boston, MA 02215, USA. Electronic mail: dkaczka@bidmc.harvard.edu atelectasis, pulmonary edema, increased lung resistance, and reduced lung compliance. Frank alveolar flooding and derecruitment reduce the effective surface area available for gas exchange. The remaining open areas are susceptible to end-inspiratory overdistention during positive pressure ventilation, since only a fraction of the injured lung is available to receive the tidal volume delivered with each breath. In addition, a portion of the ventilated region may become derecruited during expiration, leading to the generation of repetitive injurious stresses caused by cyclic re-opening during each subsequent inspiration. Over a period of time, the mechanical stresses associated with these phenomena can damage pulmonary epithelium and result in the release of inflammatory mediators that have the potential to worsen the injury. Current clinical practice seeks to limit end-expiratory derecruitment through the judicious use of positive end-expiratory pressure (PEEP), as well as reduce inspiratory overdistention with low tidal volumes. ${ }^{1}$ Mechanical heterogeneity in the injured lung is thus a central feature of the pathophysiology of ALI, ${ }^{3,4,21}$ and its accurate assessment may be extremely valuable for patient management. ${ }^{12}$

High-resolution computed tomography $(\mathrm{CT})$ provides the most detailed structural information on the lung, and demonstrates that heterogeneity of regional aeration and derecruitment depends on disease severity, tidal volume, and PEEP. ${ }^{11}$ More recently, other investigators have demonstrated that parenchymal tissue deformation determined from $\mathrm{CT}$ image registration may provide an anatomic map of regional parenchymal compliance. ${ }^{8,14,29}$ Practical issues, however, make CT unsuitable for the frequent monitoring at the bedside for ongoing ventilatory management.

Respiratory mechanical impedance $\left(Z_{\mathrm{rs}}\right)$, on the other hand, can be measured as frequently as needed 
using equipment that is readily adapted to the ICU setting. ${ }^{20}$ Previous experimental and modeling studies from our group have demonstrated a strong association between changes in $Z_{\mathrm{rs}}$ and mechanical heterogeneity of the injured lungs. ${ }^{21,22}$ Furthermore, the magnitude and distribution of parenchymal stiffness can be estimated from the frequency-dependence of $Z_{\mathrm{rs}}$, thus providing a simple and noninvasive means to quantify 'functional' heterogeneity under dynamic conditions. $^{21,22}$ However, $Z_{\mathrm{rs}}$ yields a less direct assessment of heterogeneity than that provided by CT, and there exists no information as to whether the two techniques might complement each other. ${ }^{5}$

The aim of this study was to compare the assessments of regional mechanics in a canine model of ALI using two distinct approaches: (1) quantification of parenchymal tissue compliance distributions through inverse modeling of measured $Z_{\mathrm{rs}}$; and (2) estimation of regional lung strains using $3 \mathrm{D}$ CT image registration. The authors anticipate that these results may provide new insight into the structure-function relationships of the injured lung, as well as a platform for improving and optimizing conventional or oscillatory ventilation in patients.

\section{METHODS}

\section{Animal Preparation and Measurements}

Experiments were performed in six male dogs (average weight $25.6 \pm 2.48 \mathrm{~kg}$, range $22.0-33.0 \mathrm{~kg}$ ). All protocols were approved by the Institutional Animal Care and Use Committee. After placement of a peripheral intravenous line, each dog was anesthetized with thiopental $\left(10-20 \mathrm{mg} \mathrm{kg}^{-1}\right.$ induction with $1-5 \mathrm{mg} \mathrm{kg}^{-1} \mathrm{~h}^{-1}$ maintenance infusion), orally intubated and mechanically ventilated. Muscle relaxation was achieved with vecuronium $\left(0.7-1.3 \mu \mathrm{g} \mathrm{kg}^{-1}\right.$ $\min ^{-1}$ ). Baseline $Z_{\mathrm{rs}}$ measurements were made at mean airway pressure levels of $5,10,15$, and $20 \mathrm{~cm} \mathrm{H}_{2} \mathrm{O}$ applied in random order using a custom-built servocontrolled pneumatic oscillator. ${ }^{19}$ A deep inflation to $30 \mathrm{~cm} \mathrm{H}_{2} \mathrm{O}$ for $30 \mathrm{~s}$ was performed before each $Z_{\mathrm{rs}}$ measurement, after which tracheal pressure was reduced to the specified mean airway pressure. A discretized broad-band excitation waveform with spectral energy over frequencies from 0.078 to $8.9 \mathrm{~Hz}$ was generated from a D/A board (Data Translations DT-2811, Marlboro, MA) at a rate of $40 \mathrm{~Hz}$, electronically low pass filtered at $10 \mathrm{~Hz}$ (Frequency Devices, Inc., Ottawa, IL), and presented as the driving signal to the oscillator. This signal was periodic over $25.6 \mathrm{~s}$, and was repeated 4-5 times such that each $Z_{\mathrm{rs}}$ measurement lasted approximately $2 \mathrm{~min}$. The amplitude of the excitation waveform was manually adjusted to yield peak-to-peak oscillatory tracheal pressures of $2-3 \mathrm{~cm}$ $\mathrm{H}_{2} \mathrm{O}$, resulting in oscillatory volumes of $30-50 \mathrm{~mL}$. Airway flow was measured with a calibrated pneumotachograph (Hans Rudolph, Kansas City, MO) coupled to a differential pressure transducer (Celesco LCVR 0-2 cm $\mathrm{H}_{2} \mathrm{O}$, Canoga Park, CA). Tracheal pressure was measured with a pressure transducer (Celesco LCVR 0-50 $\mathrm{cm} \mathrm{H}_{2} \mathrm{O}$ ) attached to a small polyethylene catheter extending $\sim 2 \mathrm{~cm}$ past the distal end of the endotracheal tube. The resulting pressure and flow waveforms were low pass filtered at $10 \mathrm{~Hz}$ and sampled at $40 \mathrm{~Hz}$ by an A/D board (Data Translations DT-2811). After each forced oscillation measurement, whole lung helical CT scans were obtained at the same mean airway pressure using a Sensation-16 scanner (Siemens, Iselin, NJ) during an 8 -s period of apnea at $137 \mathrm{kVp}$ and $165 \mathrm{~mA}$. The CT images were reconstructed with $2.5 \mathrm{~mm}$ slice thickness and a $512 \times 512$ matrix (voxel size $0.46 \mathrm{~mm} \times$ $0.46 \mathrm{~mm} \times 2.5 \mathrm{~mm}$ ) using $175 \mathrm{~mm}$ field at a window level of $-450 \mathrm{HU}$ and window width of $1350 \mathrm{HU}$. After these baseline measurements, each dog awoke from general anesthesia, was extubated, and was observed for several hours.

Non-survival lung injury experiments were performed in the same animals 1 week after these baseline measurements. Induction and maintenance of general anesthesia occurred as above. After insertion of femoral arterial and venous catheters, a SwanGanz catheter was positioned into the pulmonary artery, all using sterile technique. Systemic arterial, pulmonary arterial, and central venous pressures were continuously monitored. Core body temperature was maintained at $36 \pm 1{ }^{\circ} \mathrm{C}$ using radiant heat lamps. Gas exchange was assessed by measurement of arterial blood gases, and shunt fraction $\left(\dot{Q}_{\mathrm{S}} / \dot{Q}_{\mathrm{T}}\right)$ was determined using standard equations. ${ }^{26}$ Lung injury was induced with an infusion of oleic acid $\left(0.08 \mathrm{cc} \mathrm{kg}^{-1}\right.$ over $\left.20 \mathrm{~min}\right)$ into the right atrium. After maturation of the injury, defined by ratio of partial pressure of arterial oxygen to fraction of inspired oxygen $\left(\mathrm{P}_{\mathrm{a}} \mathrm{O}_{2} / \mathrm{F}_{\mathrm{i}} \mathrm{O}_{2}\right)$ being less than $300, Z_{\mathrm{rs}}$ measurements and $\mathrm{CT}$ scans were obtained as above. At the conclusion of the lung injury experiment, each animal was euthanized with an intravenous overdose of pentobarbital (10-20 mg kg-1) followed by rapid injection of $10-15 \mathrm{~mL}$ saturated $\mathrm{KCl}$ into the right atrium.

\section{Respiratory Impedance Analysis}

The $Z_{\mathrm{rs}}$ as a function of angular frequency $(\omega)$ was computed for each condition from the sampled oscillatory pressure and flow waveforms using a Welch 
overlap-average periodogram technique with a 25.6-s rectangular window and $80 \%$ overlap. ${ }^{34}$ Each timedomain record was visually inspected to ensure no apparent distortions occurred in the pressure or flow oscillations, indicating the presence of nonlinear phenomena such as intracycle derecruitment or flow-limitation. $^{20}$ After neglecting the first 500 sampled data points $(\sim 12.5 \mathrm{~s})$ to minimize transient effects, $12-20$ overlapping windows were used to calculate $Z_{\mathrm{rs}}$ for each animal. The heterogeneous mechanical properties of the respiratory system were assessed by fitting a distributed tissue compliance model to each $Z_{\mathrm{rs}}$ spectrum $^{21}$ :

$$
\hat{Z}_{\mathrm{rs}}(\omega)=\left[\int_{C_{\min }}^{C_{\max }} \frac{P(C)}{R+j \omega I+\frac{(\eta-j)}{C \omega^{\alpha}}} d C\right]^{-1}
$$

where $R$ and $I$ represent the resistance and inertance, respectively, of the airways (although both parameters are slightly influenced by small contributions from chest wall), $\eta$ represents tissue hysteresivity, ${ }^{10}$ and $\alpha=(2 / \pi) \tan ^{-1}(1 / \eta)$. The variable $C$ accounts for distributed tissue compliances, which are assumed to be arranged in parallel and vary according to a pre-defined probability density function $P(C)$. These compliances are linked to $\eta$ through the "constant-phase" behavior of the respiratory system. ${ }^{15}$ As in previous studies by this author group, ${ }^{21,22} P(C)$ was allowed to follow either hyperbolic, uniform, or linear forms. In addition, a peaked triangular distribution function was also developed to give a closer approximation to the compliance distributions obtained from our image registration technique (see below). In all the cases, $P(C)$ was bounded by upper and lower values $C_{\min }$ and $C_{\max }$, respectively. For each of the four $P(C)$ distribution functions, closed-form expressions for Eq. (1) were obtained using an open source symbolic manipulator (wxMaxima 0.8.1, http://wxmaxima.sourceforge.net), and the five independent model parameters $(R, I, \eta$, $C_{\min }$, and $C_{\max }$ ) were estimated using an unconstrained nonlinear gradient search technique (Matlab, The Matworks, Inc. Natick, MA). The model with the "best" distribution function was determined from the $P(C)$ yielding the lowest sum-of-square differences. From this, the authors determined the "effective" or average tissue compliance $(\bar{C})$ and the heterogeneity of tissue compliances $\left(\sigma_{C}\right)$ as:

$$
\bar{C}=\int_{C_{\min }}^{C_{\max }} C P(C) d C ; \quad \sigma_{C}=\sqrt{\int_{C_{\min }}^{C_{\max }}(C-\bar{C})^{2} P(C) d C}
$$

The coefficient of variation for regional compliance was determined as $C V_{C}=100 \% \times \sigma_{C} / \bar{C}$.

\section{Image Processing and Registration}

The CT images were first analyzed by segmenting the lung tissue contained within the pleural membrane from chest wall and mediastinum using a semi-automated thresholding process. ${ }^{16}$ Each segmented image was then visually inspected to ensure anatomic accuracy and was manually corrected for any errors due to the difficulty of segmenting high-density flooded regions. Total lung volume $\left(V_{\mathrm{TOT}}\right)$ at each inflation pressure was determined from the sum of all individual voxel volumes contained within the entire segmented lung. The total compliance from one pressure to the next was computed from the segmented lung scans as the change in volume divided by the corresponding change in airway pressure $\left(C_{\mathrm{TOT}}^{\mathrm{SEG}}=\Delta V_{\mathrm{TOT}} / \Delta P_{\mathrm{ao}}\right)$. Next, rigid alignment of the segmented scans was performed, and Hounsfield unit density was converted to 8-bit gray scale. The resulting transverse images were then resized by half, with the size of each voxel increasing to $0.92 \mathrm{~mm} \times 0.92 \mathrm{~mm} \times 2.5 \mathrm{~mm}$. Estimates of regional compliances were obtained using an image registration algorithm in which a 3D warping function mapped individual lung volume elements at a specified inflation pressure to corresponding volume elements at the next highest inflation pressure based on surface features and local intensity differences. ${ }^{7,18,29}$

With this algorithm, an individual voxel element $n$ located at coordinates $(x, y, z)$ at one specified inflation pressure is assumed to be displaced to a new location $(u, v, w)$ at the next higher inflation pressure. The regional deformation of this element can thus be quantified from its Jacobian matrix $\mathbf{J}_{\mathbf{n}}$, which contains the partial derivatives defining its differential expansion or contraction from one pressure state to the next ${ }^{6,8,29}$ :

$$
\mathbf{J}_{\mathbf{n}}=\left[\begin{array}{ccc}
1+\frac{\partial u}{\partial x} & \frac{\partial u}{\partial y} & \frac{\partial u}{\partial z} \\
\frac{\partial v}{\partial x} & 1+\frac{\partial v}{\partial y} & \frac{\partial v}{\partial z} \\
\frac{\partial w}{\partial x} & \frac{\partial w}{\partial y} & 1+\frac{\partial w}{\partial z}
\end{array}\right]
$$

This $\mathbf{J}_{\mathbf{n}}$ provides an estimate of the directional tissue strains for the element $n$, and thus allows for the determination of a regional deformation field with voxel-scale resolution. The determinant $\left|\mathbf{J}_{\mathbf{n}}\right|$ is a scalar quantity that can be interpreted as a ratio between the two volume states:

$$
\left|\mathbf{J}_{\mathbf{n}}\right|=\frac{V_{n}+\Delta V_{n}}{V_{n}}
$$

where $V_{n}$ is the volume of voxel element $n$, and $\Delta V_{n}$ is its change in volume at the higher inflation pressure. Thus, in this analysis, the authors determined the percentage of lung expansion and contraction based on the fraction of those $\left|\mathbf{J}_{\mathbf{n}}\right|$ greater than or less than one, respectively. 
The authors determined a local compliance $C_{n}$ for each element $n$ in the registered lung fields by rearranging Eq. (4) to solve for $\Delta V_{n}$ and dividing by the overall transrespiratory pressure change $\Delta P_{\mathrm{ao}}$ :

$$
C_{n}=\frac{\Delta V_{n}}{\Delta P_{\mathrm{ao}}}=\frac{V_{n}}{\Delta P_{\mathrm{ao}}}\left(\left|\mathbf{J}_{\mathbf{n}}\right|-1\right) .
$$

Summing Eq. (5) across all $n$ for the registered image yielded the Jacobian-based estimate for total respiratory compliance, $C_{\mathrm{TOT}}^{\mathrm{JAC}}$ :

$$
C_{\mathrm{TOT}}^{\mathrm{JAC}}=\sum_{n=1}^{N} C_{n}=\sum_{n=1}^{N} \frac{V_{n}}{\Delta P_{\mathrm{ao}}}\left(\left|\mathbf{J}_{\mathbf{n}}\right|-1\right)
$$

which can then be compared to the total compliance obtained from image segmentation. Thus, as a check for internal consistency of these image analyses, the authors examined the relationship between $C_{\mathrm{TOT}}^{\mathrm{JAC}}$ and $C_{\mathrm{TOT}}^{\mathrm{SEG}}$ across all dogs at baseline and after lung injury at each inflation pressure pair (Fig. 1). The authors found a significant correlation between these two variables $\left(r^{2}=0.78, p<0.01\right)$, with a regression line almost indistinguishable from the line of identity. However, the authors also observed considerably more scatter in these data at higher values of compliance.

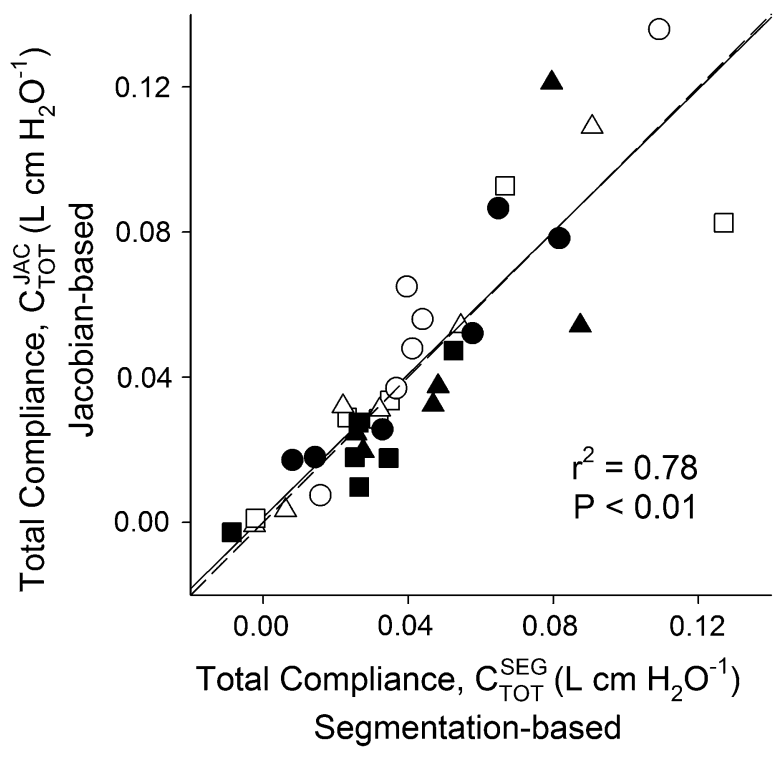

FIGURE 1. Comparison of total respiratory compliance computed from the Jacobian approach of Eq. (6) ( $C_{\text {TOT }}^{\text {JAC }}$ to the segmented image approach ( $C_{\mathrm{TOT}}^{\mathrm{SEG}}$ ) for all six dogs at baseline (white) and after lung injury (black) for pressure changes between 5-10 (circles), 10-15 (squares), and 15-20 (triangles) $\mathrm{cm} \mathrm{H}_{2} \mathrm{O}$. Linear regression yielded a significant correlation (solid line, $r^{2}=0.78, p<0.01$ ) between to two approaches which was indistinguishable from the line of identity (dashed line).

\section{Statistical Analysis}

Data for animals are reported as means \pm standard errors. One-way analysis of variance (ANOVA) was used to assess the pressure-dependence of the model parameters from Eqs. (1) and (2) as well as the means and standard deviations of the compliance distributions of Eqs. (5) and (6) before and after lung injury. If significance was obtained with ANOVA, post hoc analysis was performed using the Tukey HSD criterion. At each airway pressure, pre- and post-injury comparisons of the variables were made using twotailed paired $t$-tests. $p<0.05$ was considered statistically significant.

\section{RESULTS}

Baseline and post-injury gas exchange and hemodynamic data during conventional mechanical ventilation are shown in Table 1. After lung injury, significant decreases in $\mathrm{P}_{\mathrm{a}} \mathrm{O}_{2} / \mathrm{F}_{\mathrm{i}} \mathrm{O}_{2}$, were observed, along with significant increases in $\dot{Q}_{S} / \dot{Q}_{T}$ and mean pulmonary arterial pressure. No significant changes were observed for cardiac output or mean systemic arterial pressure after injury.

Figure 2 shows the numbers of dogs that were best fit by each of three different tissue compliance distributions, based on those models with $P(C)$ functions yielding the lowest minimum sum of squared differences between the actual $Z_{\mathrm{rs}}$ and corresponding prediction. For both baseline and injury conditions, the $Z_{\mathrm{rs}}$ for most dogs was best described by a uniform distribution of tissue compliances. Under no condition was the triangular $P(C)$ appropriate to describe $Z_{\mathrm{rs}}$.

TABLE 1. Gas exchange and hemodynamic data for the six dogs at baseline and after lung injury.

\begin{tabular}{lccc}
\hline & Baseline & Injury & Comparison \\
\hline $\mathrm{CO}\left(\mathrm{L} \mathrm{min} \mathrm{min}^{-1}\right)$ & $3.2 \pm 0.6$ & $2.4 \pm 0.5$ & $\mathrm{NS}$ \\
$\mathrm{MSAP}(\mathrm{mmHg})$ & $112 \pm 24$ & $110 \pm 12$ & $\mathrm{NS}$ \\
$\mathrm{MPAP}(\mathrm{mmHg})$ & $15 \pm 5$ & $21 \pm 6$ & $p<0.05$ \\
$\mathrm{pH}$ & $7.34 \pm 0.06$ & $7.27 \pm 0.04$ & $\mathrm{NS}$ \\
$\mathrm{P}_{\mathrm{a}} \mathrm{CO}_{2}(\mathrm{mmHg})$ & $41.53 \pm 5.85$ & $48.93 \pm 6.84$ & $\mathrm{NS}$ \\
$\mathrm{P}_{\mathrm{a}} \mathrm{O}_{2} / \mathrm{F}_{\mathrm{i}} \mathrm{O}_{2}(\mathrm{mmHg})$ & $528 \pm 37$ & $197 \pm 68$ & $p<0.001$ \\
$\dot{Q}_{\mathrm{S}} / \dot{Q}_{T}$ & $0.17 \pm 0.06$ & $0.33 \pm 0.05$ & $p<0.001$ \\
$\mathrm{Hemoglobin}(\mathrm{g} / \mathrm{dL})$ & $9.75 \pm 1.67$ & $11.10 \pm 1.24$ & $\mathrm{NS}$ \\
\hline
\end{tabular}

Data are presented as mean \pm standard error.

$\mathrm{CO}$, Cardiac output; MSAP, mean systemic arterial pressure; MPAP, mean pulmonary arterial pressure; $\mathrm{pH}$, negative logarithm of hydrogen ion concentration; $\mathrm{P}_{\mathrm{a}} \mathrm{CO}_{2}$, partial pressure of arterial carbon dioxide; $\mathrm{P}_{\mathrm{a}} \mathrm{O}_{2} / \mathrm{F}_{\mathrm{i}} \mathrm{O}_{2}$, ratio of partial pressure of arterial oxygen to fraction of inspired oxygen; $\dot{Q}_{S} / \dot{Q}_{T}$, shunt fraction. 


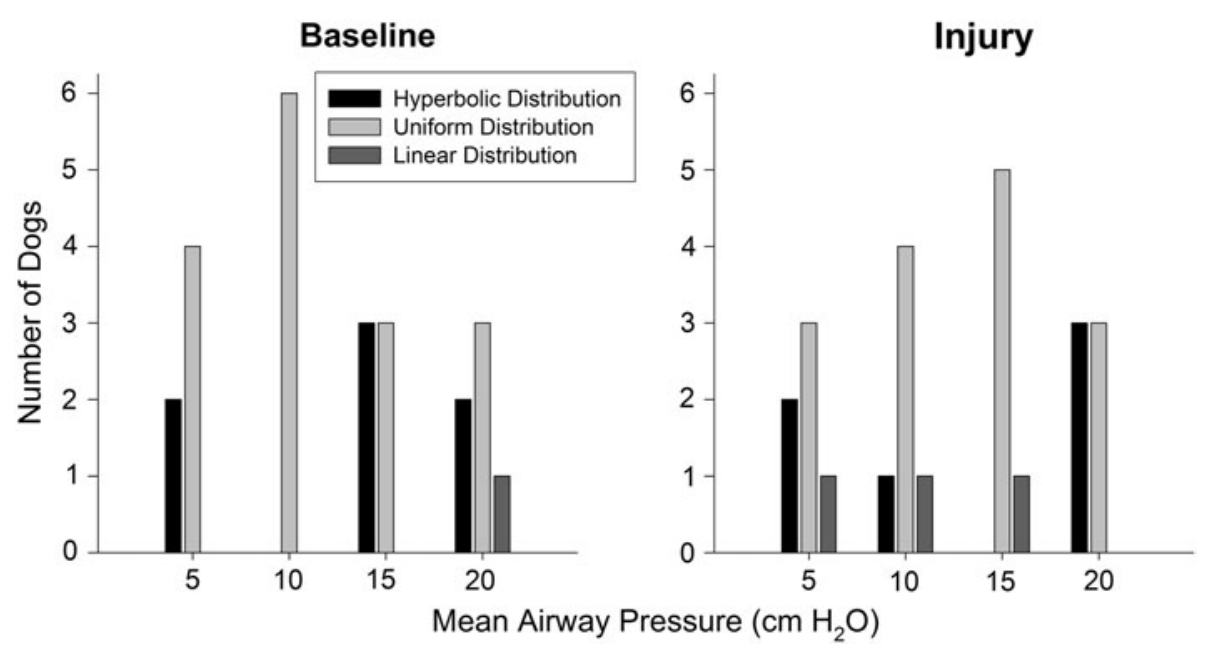

FIGURE 2. Best-fit tissue compliance distributions obtained for the six dogs studied at baseline and after lung injury at four different mean airway pressures. See text for details.

Figure 3 summarizes the distributed tissue model parameters $R, I, \eta, \bar{C}, \sigma_{C}$, and $C V_{C}$ obtained from the $Z_{\mathrm{rs}}$ spectra for mean airway pressures of $5,10,15$, and $20 \mathrm{~cm} \mathrm{H} \mathrm{H}_{2} \mathrm{O}$. Shown are the average model parameters obtained for the six dogs at baseline and after lung injury, assuming the $P(C)$ for each dog yielding the best fit to the $Z_{\mathrm{rs}}$ data. Under baseline conditions, $\bar{C}$ decreased significantly by ANOVA with increasing airway pressure. No clear pressure-dependent trends were observed for any other variable at baseline, although both $\eta$ and $\sigma_{C}$ tended to be lower at higher airway pressures. After ALI, both $R$ and $I$ were significantly increased compared to baseline at almost all distending pressures, while $\bar{C}$ was significantly decreased. In addition, the post-injury $R$ demonstrated pronounced negative dependence on airway pressure. The $C V_{C}$ parameter tended to increase after ALI, with its highest value occurring at $10 \mathrm{~cm} \mathrm{H}_{2} \mathrm{O}$.

Based on the segmented CT scans obtained at each inflation pressure, the authors determined total lung pressure-volume curves for the six dogs. Figure $4 \mathrm{a}$ summarizes these data for all dogs at baseline and after lung injury. For either condition, the pressurevolume relationship was fairly linear over the $5-20 \mathrm{~cm}$ $\mathrm{H}_{2} \mathrm{O}$ range. After injury, significant decreases in total lung volume were observed at each distending pressure compared to baseline. Figure $4 \mathrm{~b}$ summarizes $C_{\mathrm{TOT}}^{\mathrm{SEG}}$ for all six dogs. While the authors observed no significant pairwise differences between the baseline and post-injury values at any inflation pressure, postinjury $C_{\text {TOT }}^{\mathrm{SEG}}$ tended to be lowest at the $10-15 \mathrm{~cm} \mathrm{H} \mathrm{H}_{2} \mathrm{O}$ range.

Figure 5 shows example Jacobian determinant images based on registration in transverse and coronal sections for a representative dog at baseline and after lung injury for inflation pressures pairs 5-10, 10-15, and $15-20 \mathrm{~cm} \mathrm{H}_{2} \mathrm{O}$. Under baseline conditions at the lower inflation pressure of $5-10 \mathrm{~cm} \mathrm{H}_{2} \mathrm{O}$, the lungs exhibited fairly uniform expansion throughout the transverse section, although the registration algorithm did predict small regions of compression occurring near the mediastinum. Over the $10-15 \mathrm{~cm} \mathrm{H}_{2} \mathrm{O}$ inflation, expansion occurred preferentially in the more dependent regions. At the highest inflation pressure between 15 and $20 \mathrm{~cm} \mathrm{H}_{2} \mathrm{O}$, additional regions of compression adjacent to the mediastinum were observed. After ALI, the expansion fields appeared to be more heterogeneous at all inflation pressures for this representative dog, with regions of relative compression interspersed throughout the transverse sections.

Based on the fraction of Jacobian determinants greater than or less than one, the percentage of lung tissue undergoing expansion or compression, respectively, was determined. A summary of percent lung volume expanded and compressed at the three different inflation pressure-pairs before and after lung injury is shown in Fig. 6. For the baseline condition, the amount of tissue compressed tended to increase with increasing inflation pressure, although this trend was not apparent after lung injury. For either condition, regions of expansion comprised $82-93 \%$ of the total change in lung volume, while regions of compression comprised $7-17 \%$.

Based on these Jacobian deformation fields, the distribution of regional compliances $\left(C_{n}\right)$ for each dog was determined by multiplying each element in the field by the voxel size and normalizing by the change in pressure according to Eq. (5). Figure 7 shows these distributions (in units of $\mu \mathrm{lcm} \mathrm{H}_{2} \mathrm{O}^{-1}$ ) throughout the entire lung for the same representative dog as in Fig. 5 

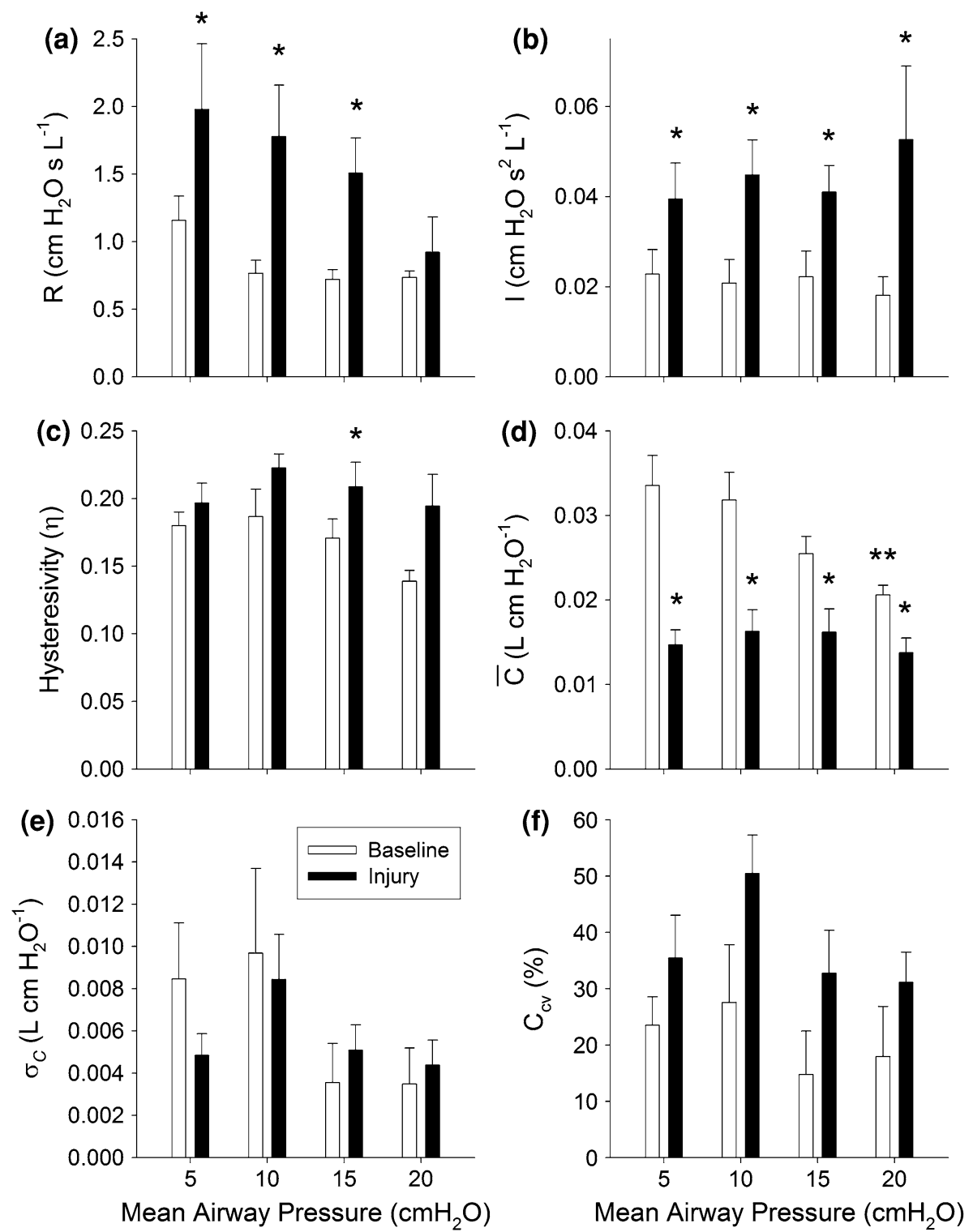

FIGURE 3. Summary of distributed tissues model parameters: (a) $R$, (b) $l$, (c) $\eta$, (d) $\bar{C}$, (e) $\sigma_{C}$, and (f) coefficient of tissue variation $C V_{C}=\sigma_{C} / \bar{C}$ vs. mean airway pressure for all six dogs. Data are presented as means \pm standard errors at baseline (white) and after lung injury (black), assuming the $P(C)$ for each dog yielding the best fit to the $Z_{\mathrm{rs}}$ data. *Significantly different from baseline data at same mean airway pressure using two-tailed paired $t$ test. ${ }^{*}$ Significantly different from corresponding data at 5 and $10 \mathrm{~cm} \mathrm{H}_{2} \mathrm{O}$ under same condition using ANOVA and Tukey HSD criterion $(p<0.05)$.

at the three different inflation pressure pairs. Under baseline conditions, the regional compliance distributions followed a unimodal pattern, although after ALI, the distributions at the lower inflation pressures became somewhat bimodal for this dog. Note that negative values of regional compliance correspond to regions that were compressed during the inflation. The greatest amount of compression occurred at the lowest inflation pressures after lung injury (i.e., $5-10 \mathrm{~cm}$ $\mathrm{H}_{2} \mathrm{O}$ ).

Figure 8 summarizes the means and standard deviations of the regional compliance distributions for all the six dogs as computed from the registered images according to Eq. (5). Also shown are the total respiratory compliances as determined from the sum of the individual regional compliance values according to Eq. (6). Under baseline conditions, both the mean regional compliance and total compliance decreased with increasing inflation pressure, similar to the mean compliance estimated using forced oscillations $(\bar{C}$, Fig. 2). The baseline standard deviation of compliance was relatively constant with inflation pressure, suggesting that the mechanical heterogeneity of the lung tissues was relatively stable over this pressure range 

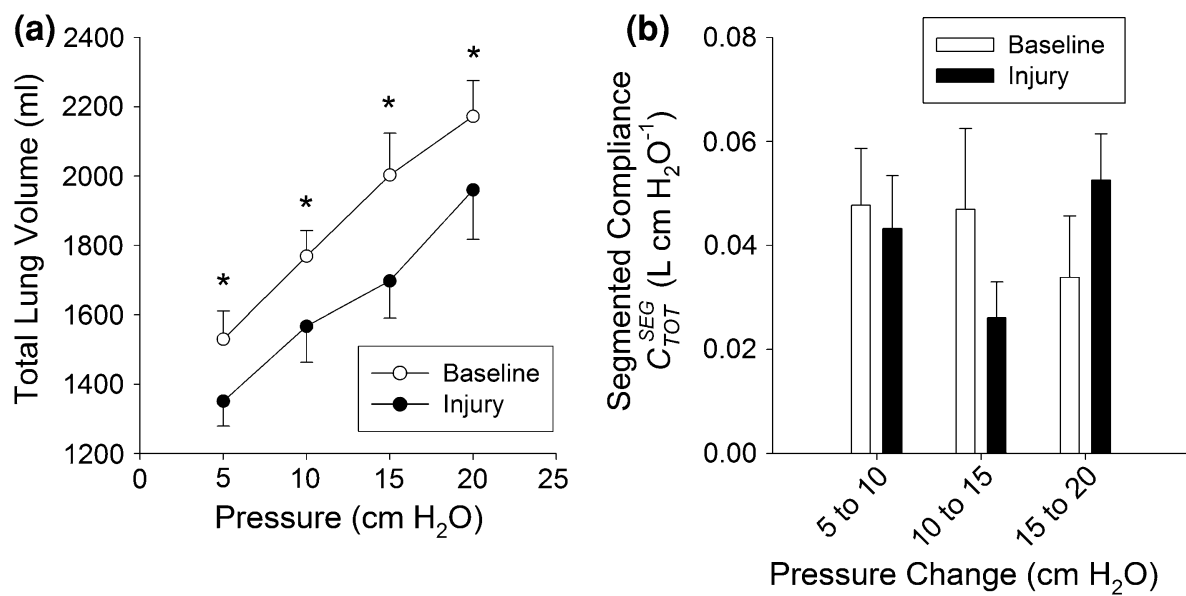

FIGURE 4. (a) Total segmented lung volume vs. mean airway pressure at baseline (white) and after lung injury (black). *Significantly higher from baseline data at same mean airway pressure using two-tailed paired $t$ test $(p<0.05)$. (b) Total segmented compliance $\left(C_{\mathrm{TOT}}^{\mathrm{SEG}}\right)$ vs. inflation pressure at baseline (white) and after lung injury (black).

(Fig. 8b). Over the $5-10$ and $10-15 \mathrm{~cm} \mathrm{H}_{2} \mathrm{O}$ pressure changes, these standard deviations tended to be increased after lung injury, although no pairwise significant changes were observed in any of these imagebased post-injury estimates of regional or total lung compliance compared to baseline. However, postinjury mean regional compliance, standard deviation of regional compliances, and total respiratory system compliance attained their minima at the moderate inflation of $10-15 \mathrm{~cm} \mathrm{H}_{2} \mathrm{O}$.

\section{DISCUSSION}

In this study, functional and structural estimates of regional lung mechanics were obtained in dogs using the oleic acid model of ALI. Techniques which can accurately quantify the heterogeneity of such mechanics may provide insight into regional derecruitment, alveolar flooding, and overdistention, all of which have been linked to ventilator-associated lung injury and may change over time as the pathology evolves. ${ }^{33}$ Our functional assessment of regional mechanics was based on forced oscillatory measurements of respiratory input impedance, $Z_{\mathrm{rs}}$, combined with an inverse modeling approach to account for distributed tissue compliances., ${ }^{3,17,21,22}$ For structural assessment, the authors relied on 3D CT image registration, a process by which in vivo anatomic estimates of regional parenchymal strains and deformations may be obtained at voxel-scale resolution. ${ }^{7,13,29}$ Before the results obtained from these two distinct approaches are compared; however, it is important to review those physiologic processes that each technique is best suited to measure, as well as their corresponding limitations.
Forced oscillatory measurements of $Z_{\mathrm{rs}}$ quantify the energy dissipation and storage behavior largely of those portions of the lung that are in direct communication with the airway opening, as well as the geometric and mechanical constraints of the chest wall. This behavior arises from complex resistive, elastic, and inertial interactions of the respiratory system, which are encapsulated in the parameter values of the model described by Eq. (1). Derecruited and flooded lung regions have very large local impedances, and their elastic contributions are effectively removed from communication with the airway opening. Moreover, the model-based approach inherent in Eq. (1) assumes that only one compliance distribution among the four studied (hyperbolic, uniform, linear, or triangular) was most suited to describe a particular $Z_{\mathrm{rs}}$ data set. ${ }^{22}$ This limits the types of compliance distributions to relatively simple closed forms that may or may not correspond to actual distributions in vivo. ${ }^{21}$ Finally, while this modeling approach may provide some quantitative insight into the nature of regional lung mechanics with only a small number of free parameters ${ }^{22}$ it provides no anatomic information on the distribution of such heterogeneity.

In contrast, image registration does provide such information by aligning three dimensional CT scans at different inflation pressures and using lung structural features to map specific regions of segmented volumes between image pairs. ${ }^{7}$ This allows for precise localization of regional expansion, contraction, and parenchymal strain. ${ }^{8,29}$ Also included in such registered volumes are non-aerated portions such as vasculature structures and derecruited/flooded regions displaced during inflation. While such regions will contribute to the estimated strain fields and local 
(a) 5 to $10 \mathrm{~cm} \mathrm{H}_{2} \mathrm{O}$
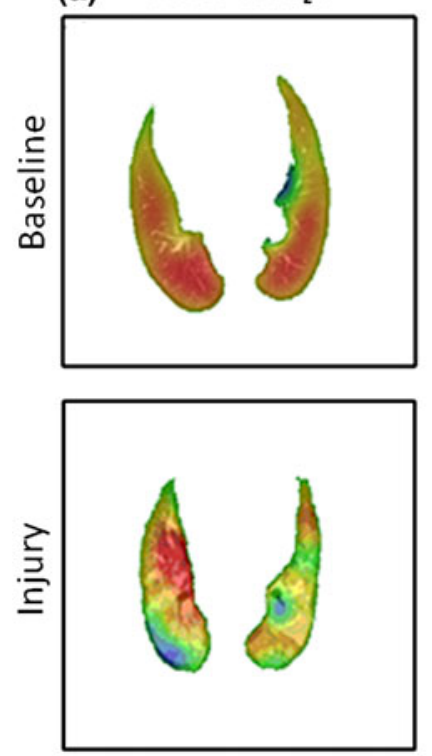

(b)
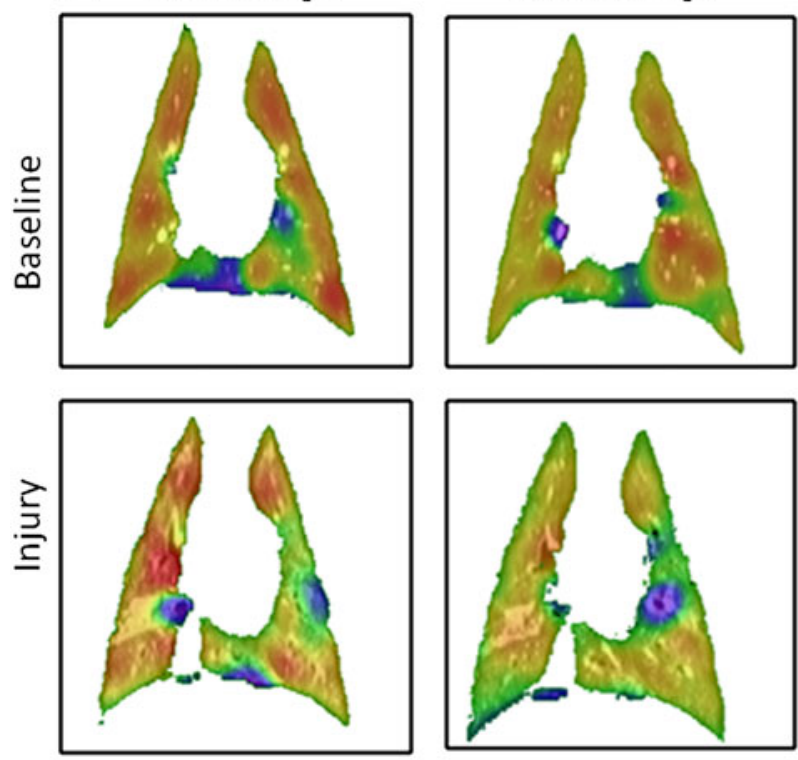

Coronal

10 to $15 \mathrm{~cm} \mathrm{H}_{2} \mathrm{O}$

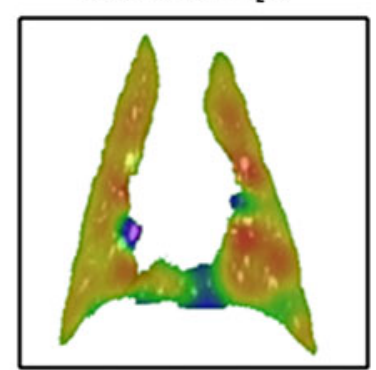

15 to $20 \mathrm{~cm} \mathrm{H}_{2} \mathrm{O}$
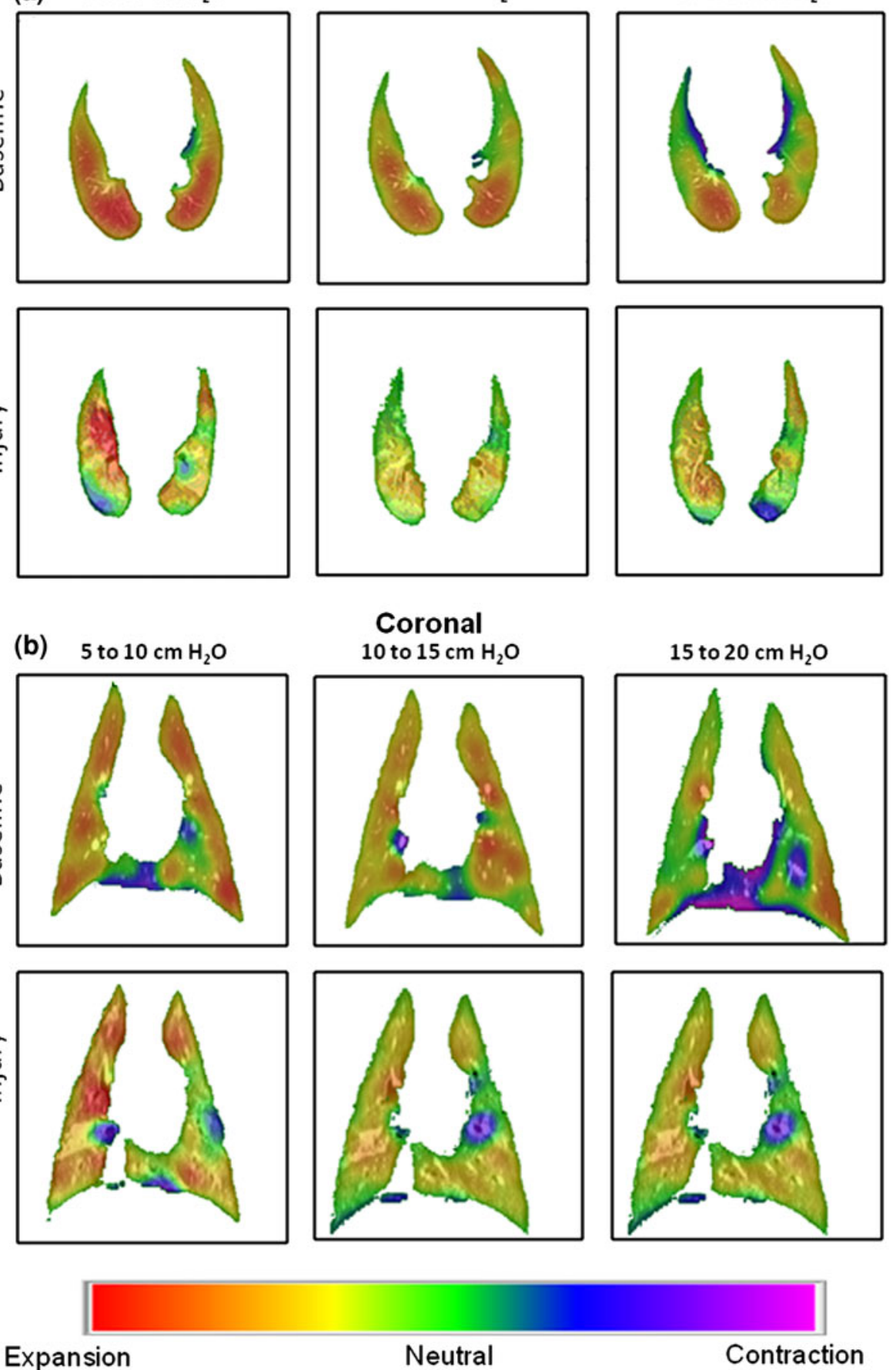

FIGURE 5. Image registration in (a) transverse and (b) coronal sections for a representative dog at baseline and after ALI for three different inflation pressures pairs. Volume changes are color coded such that yellow, orange, and red correspond to expansion, blue and purple to contraction, and green to no change in volume.

compliances determined from image registration, they will contribute only minimally to the apparent mechanical load described by $Z_{\mathrm{rs}}{ }^{27,28}$ Moreover, the image registration results were obtained under static conditions, and thus would be insensitive to the various dynamic events reflected in $Z_{\mathrm{rs}}$.

\section{Model Analysis of Respiratory Impedance}

Our assessment of respiratory mechanics using forced oscillations demonstrated that baseline estimates of average tissue compliance $\bar{C}$ decreased with increasing mean airway pressure, consistent with strain 

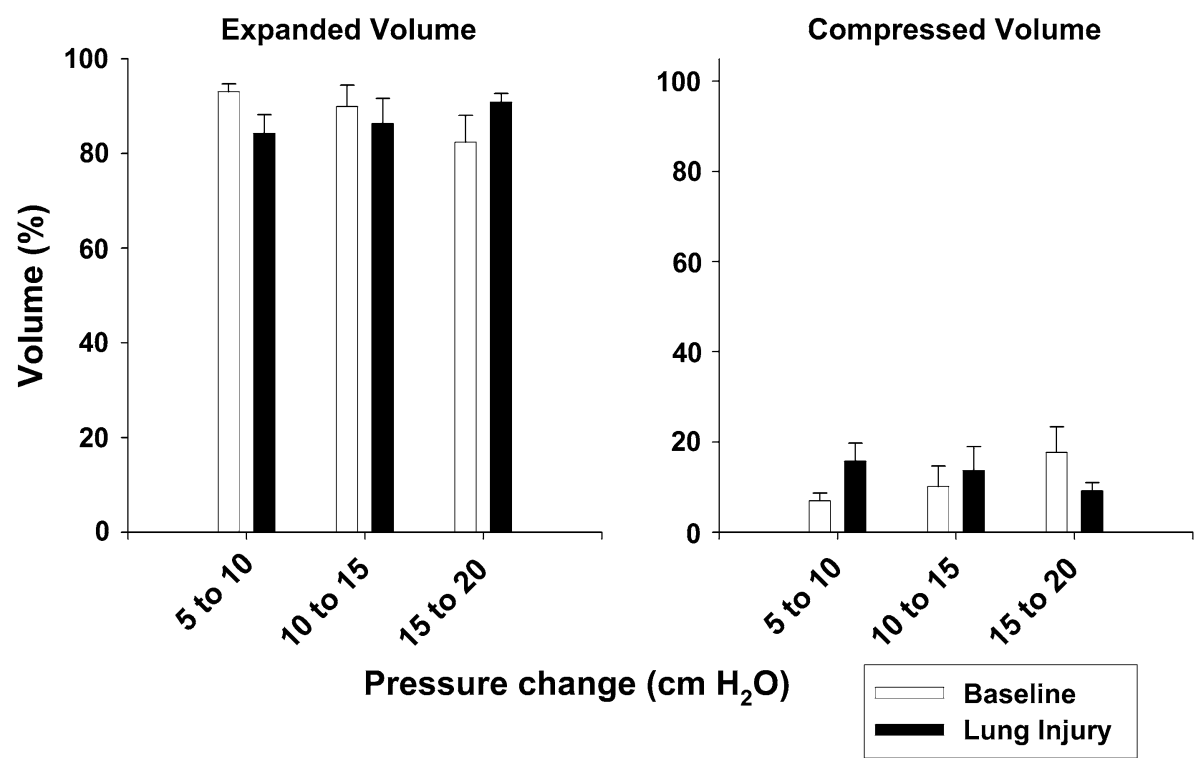

FIGURE 6. Percent of total lung volume undergoing expansion and compression at the three different inflation pressures for baseline (white) and lung injury (black) conditions as predicted from image registration. Data are presented as means \pm standard errors. Approximately $\mathbf{8 5 - 9 0 \%}$ of total lung volume expands as airway pressure increases.
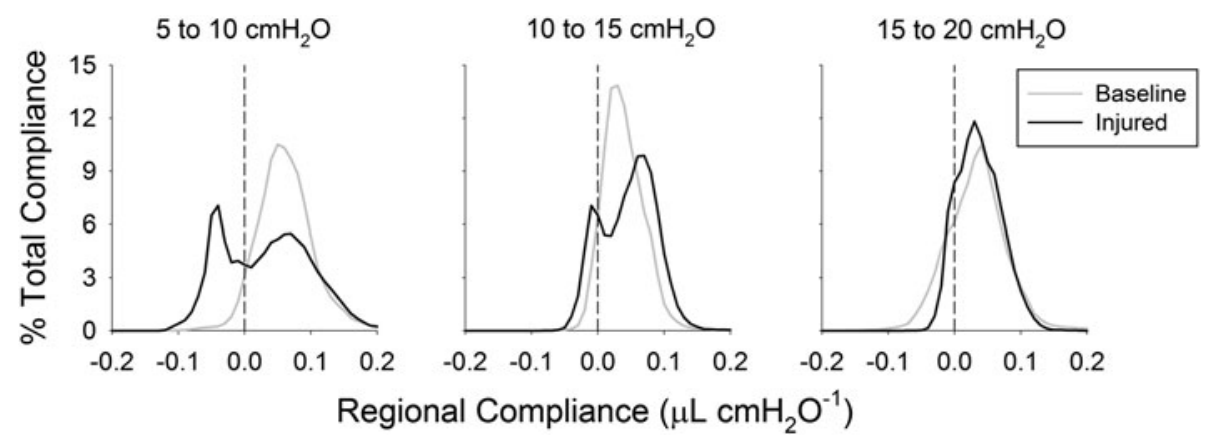

FIGURE 7. Distributions of regional compliances throughout the lung for a representative dog at three inflation pressure pairs at baseline (gray) and after lung injury (black). Dashed line denotes zero compliance.
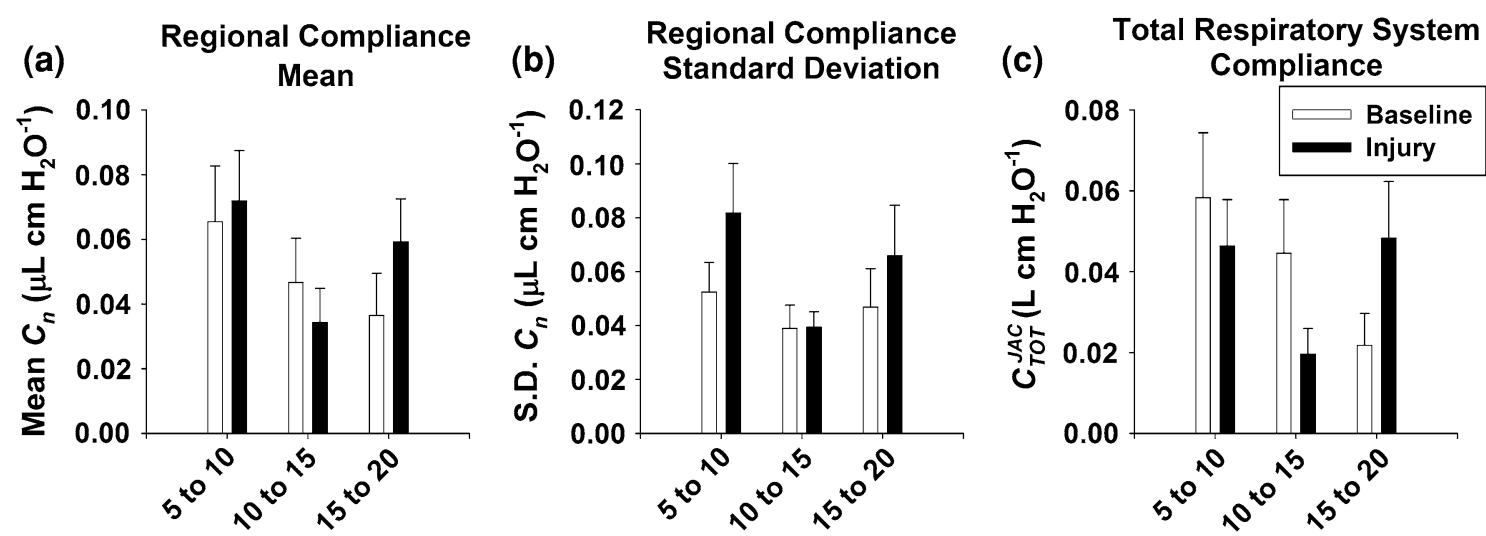

Pressure Change $\left(\mathrm{cm} \mathrm{H}_{2} \mathrm{O}\right)$

FIGURE 8. Summary of the (a) averages and (b) standard deviations of regional compliances for all the six dogs as computed from the registered images according to Eq. (5), along with (c) the total respiratory compliance as determined from Eq. (6). Data are presented as means \pm standard errors. 
stiffening of the tissues (Fig. 3d). As in previous studies, our estimates of $\bar{C}$ after ALI significantly decreased compared to baseline, ${ }^{2,21,28}$ consistent with volume loss due to derecruitment, flooding, and/or surfactant inactivation. ${ }^{33}$ However these post-injury estimates of $\bar{C}$ demonstrated no dependence on mean airway pressure. Moreover, the model-based estimates of hysteresivity (Fig. 3c) and heterogeneity (Fig. 3e) were largely unaffected by injury. While our small sample size may have been underpowered to detect significant changes in these parameters, these data do suggest that the intrinsic mechanical properties of the tissues remained unchanged in this canine model of ALI. Thus, the decreases that were observed in $\bar{C}$ relative to baseline were due mostly to derecruitment of lung units in communication with the airway opening.

Note that with increases in mean airway pressure, the baseline value of $\bar{C}$ had descended almost to the same level of post-injury $\bar{C}$ by $20 \mathrm{~cm} \mathrm{H}_{2} \mathrm{O}$ (Fig. 3d). This relative constancy of $\bar{C}$ with increasing pressure after lung injury may reflect a balance between strainstiffening of the parenchyma (tending to decrease $\bar{C}$ ) and lung recruitment (tending to increase $\bar{C}$ ). The authors would, therefore, expect minimal changes in the $Z_{\mathrm{rs}}$-based estimates of aerated tissue heterogeneity after injury, as $\sigma_{C}$ demonstrates (Fig. 3e). The tendency for $C V_{C}$ to be increased by injury in our dogs was largely a result of the decrease in $\bar{C}$, since for the same standard deviation, a decrease in mean will increase the coefficient of variation. This is consistent with the highly recruitable nature of oleic acid lung injury. ${ }^{28}$ A recent study by Lorx et al. in patients with pneumonia also characterized $Z_{\mathrm{rs}}$ with a similarly distributed tissue model, ${ }^{24}$ although they reported different dependencies in effective tissue elastance with airway pressure. Thus, our results may not be generalizable to all patients with ALI, in which more diffuse alveolar damage and hyaline membrane formation may frequently be seen. ${ }^{33}$

\section{CT Analysis of Lung Mechanics}

The pressure-volume $(\mathrm{P}-\mathrm{V})$ curves that were obtained from the segmented lung images of this study demonstrated that the injured lung inflates to a lower volume for a given airway pressure compared with baseline conditions (Fig. 4a). With CT imaging, the entire lung volume (i.e., both aerated and flooded/ derecruited regions) is measured while holding airway pressure constant. The fact that less volume at a given pressure was measured indicates that the $\mathrm{P}-\mathrm{V}$ curve has shifted to the right after injury, consistent with previous studies that construct $\mathrm{P}-\mathrm{V}$ curves from segmented $\mathrm{CT}$ images rather than measuring recoil pressure of aerated tissue. ${ }^{9,23,25}$ Note that the measurements of this study were made over the $5-20 \mathrm{~cm}$ $\mathrm{H}_{2} \mathrm{O}$ pressure range, which is arguably the most linear region of the PV curve. ${ }^{32}$ Thus, discrepancies between the $\mathrm{P}-\mathrm{V}$ slopes of healthy and injured lungs closer to TLC (i.e., 35-40 $\mathrm{cm} \mathrm{H}_{2} \mathrm{O}$ ) may not be apparent from these data.

Our image registrations indicate that the local compliances $C_{n}$ computed from the Jacobian analysis may by either unimodally or bimodally distributed (Fig. 7). It should be noted however, that $C_{n}$ is assumed to be a surrogate for regional stress-strain behavior of the lung tissues, although it is approximated as the local Jacobian-based volume change per overall transrespiratory pressure change (Eq. (5)). In reality, determination of true regional compliance requires knowledge of the regional transpulmonary pressure gradients. Thus, the $C_{n}$ that was estimated in this study might be more akin to a "transfer" compliance. Nonetheless, when all $C_{n}$ contained within the registered lung field are summed according to Eq. (6), a value for total compliance in close agreement with that obtained from the ratio of total segmented volume to airway pressure change was obtained, even for very low values of compliance (Fig. 1). The authors did observe increased variability between $C_{\mathrm{TOT}}^{\mathrm{JAC}}$ and $C_{\mathrm{TOT}}^{\mathrm{SEG}}$ for higher values of compliance, possibly due to registration error. ${ }^{29}$ Also note that computing total compliance based on the sum of $C_{n}$ assumes that all the regional compliances are independent of each other, similar to the model of Eq. (1). Thus, neither our modeling approach nor our image registration technique explicitly incorporates mechanical interdependence between lung units.

An unexpected finding is that our image registrations predict that portions of the lung are compressed during inflation, corresponding to regions of negative compliance. In transverse section, the regions of compression largely occurred adjacent to the mediastinum and chest wall (Fig. 5a), suggesting that such portions may have been forced against these structures by the underlying parenchyma as it expanded. Note that the whole-lung scans for this study were obtained during static breath-holds, and not gated to the heart rate during these periods of apnea. Such cardiac motion may certainly have resulted in partial volume artifacts or contributed to registration errors near the mediastinum. In addition, some regions of compression were observed in proximity to the diaphragm-abdomen in coronal section (Fig. 5b), raising the possibility that registration error may also have occurred near the segmented boundaries of the lung due to the difficulties of registering flooded regions that have high CT density and a relative lack of distinguishing features. Nonetheless, these findings are consistent with recent 
esophageal balloon measurements of pleural pressures in excess of alveolar pressure in some patients with ALI, indicating the compression of trapped gas behind closed airways. $^{30,31}$ The amount of compressed lung tissue estimated was not negligible $(\sim 10 \%)$, and was similar in quantity for both baseline and injured conditions (Fig. 6). This further supports the notion that the increased strain field complexity observed in Fig. 5 is due to recruitment in the injured lung, as this complexity occurs internally to the compressed borders.

\section{Comparison of Forced Oscillations and CT Image Registration}

The pressure-dependent trend of mean regional compliance obtained under baseline conditions using image registration (Fig. 8a) was similar to the more significant pressure-dependence of the $Z_{\mathrm{rs}}$-based compliance (Fig. 3d). However the parameters reflecting heterogeneity of compliance provided by the two approaches exhibit rather different dependencies on pressure (Fig. 3e vs. Fig. 8b). This presumably reflects the particular physiologic processes to which each technique is sensitive. For example, our $Z_{r \mathrm{~s}}$-based estimates of tissue compliance were obtained during dynamic oscillations in volume, and thus potentially reflect time-dependent phenomena such as stressrelaxation or pendelluft. Of course, cyclic recruitment and derecruitment during forced oscillations could potentially violate the requirements of system linearity and time-invariance assumed by our signal processing and modeling approaches. ${ }^{34}$ However, our peak-topeak oscillatory pressure excursions were likely small enough $\left(2-3 \mathrm{~cm} \mathrm{H}_{2} \mathrm{O}\right)$ such that periodic opening and closing of lung units during the excitations were minimal. Moreover, none of these time-dependent phenomena would be expected to influence compliance estimates obtained with image registration during static breath-hold conditions and larger volume excursions.

The peaked tissue compliance distributions determined from the image registrations (Fig. 7) were fundamentally different than the distributions predicted from the model-based analyses of $Z_{\mathrm{rs}}$, most of which were uniform (Fig. 2). This is despite the fact that one of the $P(C)$ distributions, which was evaluated, had a peaked, triangular form. While this may suggest that inverse model analysis of $Z_{\mathrm{rs}}$ may not provide much more information beyond a general measure of the spread of tissue compliance, ${ }^{22}$ there may also be some differences in the way that regional mechanics are distributed between aerated and non-aerated structures, as reflected in the bimodal Jacobian-based compliance distributions observed in some dogs after injury, which became unimodal at higher pressures (Fig. 6).
The results obtained from modeling of $Z_{\mathrm{rs}}$ and registration of $\mathrm{CT}$ images are consistent with the injured lung being comprised of two compartments: one that is derecruited or flooded and one that inflates normally. For example, the effective tissue compliance estimated from $Z_{\mathrm{rs}}$ demonstrated significant differences pre- vs. post-injury (Fig. 3d), while the mean regional compliance obtained from image registration exhibited minimal change (Fig. 8a). This implies that derecruited and flooded regions have very unique contributions to the overall mechanical behavior of the lung as determined from either oscillatory flow response at the airway opening or regional parenchymal strain fields. Thus, forced oscillations and image registration provide very different, but nonetheless complimentary, information regarding pulmonary structure-function relationships and their pathologic alterations.

One of the prime motivations for pursuing this study of structure and function in ALI is to determine whether mechanical ventilation of patients can be individualized beyond the implementation of simple low-tidal volume protocols. ${ }^{1}$ In particular, the design of an optimal ventilatory regimen for a given patient will depend on the ability to obtain a sufficiently detailed assessment of the mechanical status of the lungs. For practical reasons, image registration is unlikely to serve in this regard. However, this technique has enabled us to better understand the physiologic relevance of $Z_{\mathrm{rs}}$, which does have the potential to be a routine diagnostic tool at the bedside. The measurement of $Z_{\mathrm{rs}}$ can easily be incorporated into existing ventilator platforms ${ }^{3,20}$ to provide information about lung tissue heterogeneity, recruitment, and over-distention without disrupting ventilatory support. Such information could have a significant influence on the choice of tidal volumes and airway pressure ranges for individual patients. ${ }^{20}$

In summary, this study compared functional and structural estimates of regional lung mechanics at different inflation pressures in dogs with ALI using both forced oscillations and CT image registration. While $Z_{\mathrm{rs}}$ provides specific functional information regarding the heterogeneity and recruitment of injured lungs under dynamic conditions, structural assessment of regional mechanics can be obtained at voxel-level scale from the deformation field identified using CT image registration. Each technique exhibits different sensitivities to mechanical heterogeneity in this canine model of lung injury, but taken together, both provide complementary information on structure-function relationships and may allow for a more appropriate comparison of physiologic models with anatomic data. 


\section{ACKNOWLEDGMENTS}

The authors thank Beatrice Mudge, Timothy Burman, and Daniel Mulreany for their technical assistance in this study. The authors also thank Dr. Monica Hawley for her statistical analysis of the data, and Dr. Deokiee Chon for assistance with the image processing. Animal experiments were performed at the Johns Hopkins University Anesthesiology Research Labs under research protocol DO07M176. This study was supported by the NIH Grants HL089227, HL075593, HL064368, and HL079406.

\section{OPEN ACCESS}

This article is distributed under the terms of the Creative Commons Attribution Noncommercial License which permits any noncommercial use, distribution, and reproduction in any medium, provided the original author(s) and source are credited.

\section{REFERENCES}

${ }^{1}$ The ARDS Network. Ventilation with lower tidal volumes as compared with traditional tidal volumes for acute lung injury and the acute respiratory distress syndrome. N. Engl. J. Med. 342:1301-1308, 2000.

${ }^{2}$ Barnas, G. M., J. Sprung, R. Kahn, P. A. Delaney, and M. Agarwal. Lung tissue and airway impedance during pulmonary edema in the normal range of breathing. $J$. Appl. Physiol. 78:1889-1897, 1995.

${ }^{3}$ Bellardine, C., E. P. Ingenito, A. Hoffman, F. Lopez, W. Sanborn, B. Suki, and K. R. Lutchen. Heterogeneous airway versus tissue mechanics and their relation to gas exchange function during mechanical ventilation. Ann. Biomed. Eng. 33:626-641, 2005.

${ }^{4}$ Bellardine-Black, C. L., A. M. Hoffman, L. Tsai, E. P. Ingenito, B. Suki, D. W. Kaczka, B. A. Simon, and K. R. Lutchen. Relationship between dynamic respiratory mechanics and disease heterogeneity in sheep lavage injury. Crit. Care Med. 35:870-878, 2007.

${ }^{5}$ Bellardine-Black, C. L., A. M. Hoffman, L. W. Tsai, E. P. Ingenito, B. Suki, D. W. Kaczka, B. A. Simon, and K. R. Lutchen. Impact of positive end-expiratory pressure during heterogeneous lung injury: insights from computed tomographic image functional modeling. Ann. Biomed. Eng. 36:980-991, 2008.

${ }^{6}$ Castillo, R., E. Castillo, J. Martinez, and T. Guerrero. Ventilation from four-dimensional computed tomography: density versus Jacobian methods. Phys. Med. Biol. 55: 4661-4685, 2010.

${ }^{7}$ Christensen, G. E., and H. J. Johnson. Consistent image registration. IEEE Trans. Med. Imaging 20:568-582, 2001. ${ }^{8}$ Christensen, G. E., J. H. Song, W. Lu, I. El Naqa, and D. A. Low. Tracking lung tissue motion and expansion/ compression with inverse consistent image registration and spirometry. Med. Phys. 34:2155-2163, 2007.

${ }^{9}$ Downie, J. M., A. J. Nam, and B. A. Simon. Pressurevolume curve does not predict steady-state lung volume in canine lavage lung injury. Am. J. Respir. Crit. Care Med. 169:957-962, 2004.

${ }^{10}$ Fredberg, J. J., and D. Stamenovic. On the imperfect elasticity of lung tissue. J. Appl. Physiol. 67:2408-2419, 1989.

${ }^{11}$ Gattinoni, L., P. Pelosi, S. Crotti, and F. Valenza. Effects of positive end-expiratory pressure on regional distribution of tidal volume and recruitment in adult respiratory distress syndrome. Am. J. Respir. Crit. Care Med. 151:1807-1814, 1995.

${ }^{12}$ Grasso, S., T. Stripoli, M. Sacchi, P. Trerotoli, F. Staffieri, D. Franchini, V. De Monte, V. Valentini, P. Pugliese, A. Crovace, B. Driessen, and T. Fiore. Inhomogeneity of lung parenchyma during the open lung strategy: a computed tomography scan study. Am. J. Respir. Crit. Care Med. 180:415-423, 2009.

${ }^{13}$ Guerrero, T., G. Zhang, T. C. Huang, and K. P. Lin. Intrathoracic tumour motion estimation from CT imaging using the 3D optical flow method. Phys. Med. Biol. 49:4147-4161, 2004.

${ }^{14}$ Guerrero, T., R. Castillo, K. Sanders, R. Price, R. Komaki, and D. Cody. Novel method to calculate pulmonary compliance images in rodents from computed tomography acquired at constant pressures. Phys. Med. Biol. 51:11011112, 2006.

${ }^{15}$ Hantos, Z., B. Daroczy, B. Suki, S. Nagy, and J. J. Fredberg. Input impedance and peripheral inhomogeneity of dog lungs. J. Appl. Physiol. 72:168-178, 1992.

${ }^{16} \mathrm{Hu}$, S., E. A. Hoffman, and J. M. Reinhardt. Automatic lung segmentation for accurate quantitation of volumetric X-ray CT images. IEEE Trans. Med. Imaging 20:490-498, 2001.

${ }^{17}$ Ito, S., E. P. Ingenito, S. P. Arold, H. Parameswaran, N. T. Tgavalekos, K. R. Lutchen, and B. Suki. Tissue heterogeneity in the mouse lung: effects of elastase treatment. J. Appl. Physiol. 97:204-212, 2004.

${ }^{18}$ Johnson, H. J., and G. E. Christensen. Consistent landmark and intensity-based image registration. IEEE Trans. Med. Imaging 21:450-461, 2002.

${ }^{19}$ Kaczka, D. W., and K. R. Lutchen. Servo-controlled pneumatic pressure oscillator for respiratory impedance measurements and high frequency ventilation. Ann. Biomed. Eng. 32:596-608, 2004.

${ }^{20}$ Kaczka, D. W., E. P. Ingenito, and K. R. Lutchen. Technique to determine inspiratory impedance during mechanical ventilation: implications for flow-limited patients. Ann. Biomed. Eng. 27:340-355, 1999.

${ }^{21}$ Kaczka, D. W., D. N. Hager, M. L. Hawley, and B. A. Simon. Quantifying mechanical heterogeneity in canine acute lung injury: impact of mean airway pressure. Anesthesiology 103:306-317, 2005.

${ }^{22}$ Kaczka, D. W., C. B. Massa, and B. A. Simon. Reliability of estimating stochastic lung tissue heterogeneity from pulmonary impedance spectra: a forward-inverse modeling study. Ann. Biomed. Eng. 35:1722-1738, 2007.

${ }^{23}$ Karmrodt, J., C. Bletz, S. Yuan, M. David, C.-P. Heussel, and K. Markstaller. Quantification of atelectatic lung volumes in two different porcine models of ARDS. Br. J. Anaesth. 97:883-895, 2006.

${ }^{24}$ Lorx, A., B. Suki, M. Hercsuth, B. Szabó, I. Pénzes, K. Boda, and Z. Hantos. Airway and tissue mechanics in ventilated patients with pneumonia. Respir. Physiol. Neurobiol. 171:101-109, 2010.

${ }^{25}$ Luecke, T., J. P. Meinhardt, P. Herrmann, A. Weiss, M. Quintel, and P. Pelosi. Oleic acid vs saline solution lung 
lavage-induced acute lung injury: effects on lung morphology, pressure-volume relationships, and response to positive end-expiratory pressure. Chest 130:392-401, 2006.

${ }^{26}$ Lumb, A. B. Nunn's Applied Respiratory Physiology. Philadelphia: Elsevier, p. 520, 2005.

${ }^{27}$ Martynowicz, M. A., T. A. Minor, B. J. Walters, and R. D. Hubmayr. Regional expansion of oleic acid-injured lungs. Am. J. Respir. Crit. Care Med. 160:250-258, 1999.

${ }^{28}$ Martynowicz, M. A., B. J. Walters, and R. D. Hubmayr. Mechanisms of recruitment in oleic acid-injured lungs. J. Appl. Physiol. 90:1744-1753, 2001.

${ }^{29}$ Reinhardt, J. M., K. Ding, K. Cao, G. E. Christensen, E. A. Hoffman, and S. V. Bodas. Registration-based estimates of local lung tissue expansion compared to xenon CT measures of specific ventilation. Med. Image Anal. 12:752-763, 2008.
${ }^{30}$ Talmor, D., T. Sarge, C. R. O'Donnell, R. Ritz, A. Malhotra, A. Lisbon, and S. H. Loring. Esophageal and transpulmonary pressures in acute respiratory failure. Crit. Care Med. 34:1389-1394, 2006.

${ }^{31}$ Talmor, D., T. Sarge, A. Malhotra, C. R. O'Donnell, R. Ritz, A. Lisbon, V. Novack, and S. H. Loring. Mechanical ventilation guided by esophageal pressure in acute lung injury. N. Engl. J. Med. 359:2095-2104, 2008.

${ }^{32}$ Venegas, J. G., R. S. Harris, and B. A. Simon. A comprehensive equation for pulmonary pressure-volume curve. J. Appl. Physiol. 84:389-395, 1998.

${ }^{33}$ Ware, L. B., and M. A. Matthay. The acute respiratory distress syndrome. N. Engl. J. Med. 342:1334-1349, 2000.

${ }^{34}$ Welch, P. D. The use of fast Fourier transform for the estimation of power spectra: a method based on time averaging over short, modified periodograms. IEEE Trans. Audio Electroacoust. 15:70-73, 1967. 